\title{
DE DOCTORES Y MONSTRUOS: LA CIENCIA COMO TRANSGRESIÓN EN DR. FAUSTUS, FRANKESTEIN Y DR. JEKYLL AND MR. HYDE
}

\author{
Beatriz Villacañas
}

Universidad Complutense, Madrid.

\section{RESUMEN}

Este artículo, partiendo de la idea de que la ciencia ha sido muy a menudo uno de los temas más eminentemente literarios, de lo que se dan algunos ejemplos, explora esta relación entre los ámbitos literario y científico, la cual cobra especial intensidad en los casos extremos, trágicos, de la trasgresión científica, que analizamos aquí en tres casos absolutamente emblemáticos de la literatura inglesa: Dr. Faustus, Frankenstein y Dr. Jekyll and Mr. Hyde. Cada uno de los héroes de estas obras es un científico, a su manera, un doctor. Cada uno, impulsado por la pasión irrefrenable de la investigación científica, va más allá de los límites morales, religiosos, sociales a los que están sometidos como científicos y como humanos, lo que hace a su acción transgresora y a ellos mismos convertirse en monstruosos, separarse de la humanidad que les unía al resto de los hombres. Pero la búsqueda de estos doctores, como ha hecho evidente la literatura y como se ha intentado mostrar en estas páginas, tiene plena vigencia para la ciencia hoy día: sin ir más lejos, el debate médico del momento tiene como una de sus preocupaciones básicas la cuestión de la ingeniería genética, que despierta, tanto en los científicos como en el resto de la gente, tantas expectativas, tantas inquietudes, tantos miedos, tantas esperanzas.

\section{SUMMARY}

This article, setting out from the idea that science has very often been a fundamental concern of literature, of which several examples are given, explores the relationship between the literary and the scientific realms, which becomes especially intense in the extreme, tragical cases of scientific transgression. This latter is analysed here in the concrete cases of three emblematic works of English literature belonging to different historical periods: Dr. Faustus, Frankenstein and Dr. Jekyll and Mr. Hyde. Each of the heroes is a scientist, a doctor, in his own way, and each of them, impelled by an overwhelming scientific passion, trespasses on moral, religious and social limits imposed on them as men and as doctors. Their actions, or their very selves become monstruous in the course of their transgression, which brings punishment and tragedy. But the quest of these doctors, as literature has proven throughout the centuries, and as it has been my intention to explain, continues to be that of science today: it is particularly at the very core of medical debate nowadays, when questions such as genetic engineering awaken both in scientist and people in general all sorts of expectations, misgivings, fears and hopes. 
BEATRIZ VILLACAÑAS

\section{INTRODUCCIÓN}

En literatura, bien es sabido, todas las cuestiones relacionadas con la vida humana tienen acomodo. En numerosas ocasiones, además, son los casos extremos de dicho vivir humano los predilectos de cierta literatura, de ciertas manifestaciones o géneros como el goticismo o la tragedia. Estos casos extremos de amores tempestuosos, dolor sin paliativos, luchas titánicas o destinos ineludibles, entre tantos otros, han venido nutriendo la literatura de forma persistente a través de los siglos y, a su vez, gracias a ésta, han adquirido esa dimensión arquetípica que les hace parte de nuestra cultura colectiva. La relación simbiótica entre literatura y realidad, su mutuo enriquecimiento, lo paradójico y, sin duda, conflictivo de su larga convivencia constituye un campo de investigación tan apasionante como inabarcable. Una de las manifestaciones de la realidad humana, su deseo de saber, su aprendizaje, sus avances y hallazgos, todo eso que llamamos ciencia, nunca le ha sido ajeno a la literatura. La ciencia en sus diferentes manifestaciones ha sido el motivo principal de más de un texto literario y numerosos son los escritores para quienes la ciencia y el científico han constituido el tema principal o tema de importancia en algunas de sus obras. Honoré de Balzac, Emile Zola, Miguel de Unamuno, Pío Baroja, Wilkie Collins, G.B. Shaw, H.G. Wells o Aldous Huxley son, entre muchos otros, ejemplos de este interés de la literatura por la ciencia, del literato por el científico. El porqué de este interés no tiene respuesta fácil ni única tampoco, pero, quizá, algo que en parte lo explicaría está en que para la literatura, para el escritor, la ciencia y el científico pueden constituir alguno de esos casos extremos a los que en un principio se ha aludido. Esto no es extraño en absoluto si tenemos presente que la ciencia se mueve, se ha movido siempre, entre lo real y lo posible, sabe cómo sacar provecho, y lo saca, de sus propios avances, aplica sus hallazgos de forma que supongan beneficios, enormes beneficios algunas veces, a la humanidad. Esta capacidad de la ciencia, y de manera muy especial de la ciencia médica, para incidir de forma decisiva en la vida, no sólo hace que los mortales la necesitemos, sino también que la temamos. El médico en particular, el científico que más cerca está de los receptores de su práctica, quien tiene en sus manos el alivio del dolor físico, la curación de enfermedades, la vida o la muerte, resulta especialmente poderoso y, por tanto, especialmente respetado, necesitado, y también temido.

Es ese punto de encuentro entre la necesidad y el temor lo que otorga a la ciencia su fascinación y, por tanto, su cualidad literaria. Es muy a menudo el poder del científico, no siempre controlado ni controlable, lo que hace trascender su realidad cotidiana y le convierte en figura, en personaje. Arthur Koestler (1977:257), en The Act of Creation, ya habló del arquetipo literario del «científico loco», personaje malvado entregado de forma enfermiza a sus experimentos.

El movimiento hacia adelante del científico, su esfuerzo por convertir lo posible, lo imaginado, en realidad, en resumidas cuentas, el avance, choca a menudo con 
obstáculos de muy diversa índole. No es infrecuente que otros miembros de la comunidad científica, más conservadores, o reacios a que sus métodos aparezcan obsoletos frente a cualquier nuevo descubrimiento, se conviertan en durísimos obstáculos para determinados avances. Michael White, en un revelador estudio sobre la figura del médico en la novela de G. Eliot, Middlemarch, describe la hostilidad profesional que el joven y recién llegado Dr. Lydgate, con sus métodos innovadores, despierta en los médicos de la comunidad provinciana de Middlemarch:

\footnotetext{
Lydgate's determination to break with this policy (la sistemática prescripción de medicamentos como panacea en toda ocasión) is interpreted by the medical men of Middlemarch as casting aspersion on their practice as also indicative of the new doctors pride or passion to distinguish himself. (White 1985:331)
}

En muchos casos, asimismo, la propia sociedad puede sentir recelos ante lo nuevo, asentada como está en la confortabilidad de lo conocido. Thomas Hardy, en su novela The Woodlanders (1887/1982), muestra esto a través de la figura del Dr. Fitzpiers.

Es, sin embargo, el científico dispuesto a romper con todo en pos de su investigación, el que es capaz de transgredir determinadas normas éticas, aquél que siente la ciencia como pasión arrolladora que le hace prescindir de sus propias necesidades humanas, del contacto con los demás, del amor, del gozo de los sentidos, de todo aquello que no sea su obsesivo trabajo, aquél capaz de correr riesgos extremos y de enfrentarse al posible horror de sus inventos, en suma, el científico que, a mi entender, se ha convertido en uno de los más interesantes arquetipos literarios, quien será objeto de este estudio. En este caso, son tres personajes de la literatura inglesa: el Dr. Fausto, el Dr. Frankenstein y el Dr. Jekyll, protagonistas respectivos de Doctor Faustus, de Christopher Marlowe, Frankenstein, de Mary Shelley, y The Strange Case of Dr. Jekyll and Mr. Hyde, de R.L. Stevenson, los arquetipos del científico transgresor, objetos de temor y admiración, quienes serán, asimismo, los protagonistas de estas páginas. Los tres son grandes transgresores.

El Doctor Fausto, llevado de su amor apasionado por el saber, de su irrefrenable deseo de conocimiento, sobre todo de aquello que la mente humana, con sus limitaciones no sólo morales sino puramente fisiológicas, no puede conocer - lo que se ha venido llamando los grandes misterios - hace un pacto con el diablo, a quien vende su alma, para saberlo todo, para poderlo todo: veinticuatro años de saber y de poder, de dominio sobre los hombres. El Doctor Frankenstein, obsesionado con el misterio de la existencia, lucha denodadamente por descubrirlo, desea él también crear vida y no se detiene en sus experimentos hasta dar vida a su famosa y monstruosa criatura. El honorable Doctor Jekyll consigue, en el campo de la investigación química, un bebedizo capaz de producir absolutas transformaciones tanto psíquicas como fisiológicas, en este caso dentro de él mismo, sujeto y objeto de sus propios ensayos. Los 
tres, por tanto, se afanan por ser dioses y en este afán se percibe un progresivo abandono de su propia condición humana, un alejamiento de los demás hombres, una irreversible soledad. Grandes solitarios, en su momento fueron nuevas representaciones del mito de Prometeo, del desafío a los dioses, o si se prefiere, de desafío a las leyes en la naturaleza. Los tres, como Prometeo, fueron castigados de forma implacable. Es la suya una historia trágica, extrema y desmesurada como sus propias ambiciones. En lo extremo de su caracterización y de sus mencionadas ambiciones radica su monstruosidad, la monstruosidad entendida aquí como anomalía por exceso, como desmesura incontenible. De la relación que cada uno de los tres mantiene con la ciencia me ocuparé a continuación.

\section{DR. FAUSTUS: «MORE THAN HEAVENLY POWER PERMITS»}

En la obra teatral Dr. Faustus, considerada la primera tragedia del Renacimiento, es significativo el hecho de que su protagonista, su héroe trágico, sea un científico. Es bien cierto que la obra de Marlowe tiene como fuente una traducción inglesa del llamado Faustbuch, libro alemán basado a su vez en la historia supuestamente real, pero obviamente «literarizada» por la imaginación popular, de un nigromante que vivió en Alemania entre los años 1488 y 1541, al que se menciona en varios documentos de la época. Pero la cuestión no está en si este supuesto personaje real era o no un científico riguroso en términos modernos, un brujo, un alquimista o un practicante de las hoy llamadas pseudociencias, cuya frontera con lo que contemporáneamente llamamos ciencia era mucho más borrosa en los siglos quince, dieciséis o incluso diecinueve (recordemos la Frenología) que en la actualidad. La realidad es que el Fausto de Marlowe es presentado de igual modo como nigromante y como científico y, en calidad de tal, en términos renacentistas, dominaba las más diversas parcelas del saber: la astronomía, la química, también la alquimia y, por supuesto, la medicina.

La escena primera del primer acto nos muestra a un Fausto satisfecho de su dominio de todos los saberes, pero insatisfecho ante su limitada humanidad: él desea conocer y controlar los últimos misterios, aquello que puede hacer de él no ya un sabio, lo que ya es, sino un dios. Lo que caracteriza a Fausto es que en su calidad de hombre de ciencia sólo existe y actúa en función de ésta (sus habilidades en el campo de la magia son una muestra de lo ilícito y contra natura de su pacto), la ciencia es su única y excluyente pasión y no se le conocen otros amores, otros deseos. Su, en principio humano, ardor intelectual termina por consumir su humanidad, hace de él un monstruo en la hipertrofia de su deseo de saber, en su deseo de dominación por el saber, y su pacto con Mefistófeles, la venta de su alma, no es sino la consecuencia última de su deshumanización, de su monstruosidad. 
Fausto, a lo largo de los veinticuatro años de saber y poder pactados previamente (saber y poder que se revelan como más ilusorios que auténticos) solamente se comunica con Mefistófeles, el enviado de Lucifer. Entre los humanos no hay ni compañeros, ni amigos, ni amantes. Cuando le pide a Mefistófeles una esposa, éste le presenta, como burla suprema, a una diablesa entre fuegos artificiales. Fausto no es, por tanto, sólo un hombre de ciencia, es el arquetipo del hombre de ciencia al que la ciencia va despojando de su cualidad de hombre: un prototipo, un personaje representativo.

El castigo final de Fausto encaja perfectamente en la ortodoxia cristiana que en principio propugna la obra, en buena parte heredera de la Morality Play medieval, pero con el sufrimiento que acarrea, a su vez devuelve a Fausto parte de su humanidad perdida, le convierte en héroe trágico y fortalece su carácter representativo. La oscilación entre las perspectivas bajo las que se nos presenta Fausto, la heredera por un lado de la Morality Play medieval y, por otro, la renacentista, es algo que trata magníficamente Michael Mangan (1989) en su estudio crítico sobre la obra. Roger Shattuck, en su Conocimiento Prohibido (1998), se centra fundamentalmente en el Fausto de Goethe, autor que según él, saca al Fausto de Marlowe de la Edad Media. No obstante la evidente y poderosa alegoría medieval que late en la obra marloviana, el deseo prometéico que mueve al héroe, su «humanización» en el anhelo y en el dolor, hacen que el Fausto de Marlowe sobrepase el ámbito medieval en el que la sitúa Shattuck, y pueda considerarse una obra plenamente renacentista y moderna. ${ }^{1}$ El hombre aterrorizado que existe en el científico transgresor se nos presenta en el tormento de su hora final con una inmediatez y hondura psicológica que transcienden el simple marco alegórico.

En el transcurso de la última hora de los veinticuatro años, después de la cual la muerte del científico se hará ineludible y Lucifer reclamará su alma, vemos a Fausto presa del miedo, hombre al fin y al cabo que sabe que va a morir y que va a pagar por sus errores:

The hour strikes eleven -

Faustus: «Ah Faustus,

Now has thou but one bare hour to live,

And then you must be damned perpetually.

Stand still, you ever-moving spheres of heaven,

1 Shattuck pone de manifiesto la falta de unidad de tono del Fausto de Goethe y la incoherencia moral que supone su salvación final. El Fausto de Marlowe, por el contrario, no encuentra el perdón pese a que nos es dado conocer sus últimos sufrimientos. Si el autor de Conocimiento Prohibido hubiera visto en este último algo más de lo que él considera una «alegoría moral estereotipada» (p. 105) probablemente habría percibido a este transgresor como mucho más cercano al Frankenstein de M. Shelley (a quien compara con el Fausto del alemán) y al Dr. Jekyll de Stevenson de lo que su tiempo de composición y su herencia medieval sugieren a primera vista. 
That time may cease and midnight never come.»

The watch strikes -

«... Ah! Half and hour is past,

'Twil all be past anon.

Oh God, if thou wilt not have mercy on my soul,

Yet, for Christ's sake whose blood hath ransomed me,

Impose some end to my incessant pain.

Let Faustus live in Hell a thousand years.

A hundred thousand, and at last be saved» (1604/1972:336-7)

Fausto agoniza de terror porque es plenamente consciente de que será castigado de forma implacable por haber traspasado los límites, la frontera moral (en este caso de carácter religioso) que separa la práctica y el saber científicos permitidos y lo que no es lícito para ningún científico practicar si ello lleva consigo el abandono de lo humano. Ante su trágico destino, Fausto se lamenta de no haber sido en vez de hombre, cualquier criatura sin alma (él, tan apasionado por el saber) o, ya que tiene alma, se queja con desesperación de que ésta sea inmortal. Porque como él mismo reconoce, «No end is limited to damned souls» (1604/1972:337).

Es la del doctor Fausto una historia trágica, tal y como explicita el título completo que da Marlowe a su obra dramática: The Tragical History of Doctor Faustus. Su castigo final, que se presenta como ejemplar, es el que le espera al transgresor, quien siendo superior a los demás hombres, en este caso gracias a sus conocimientos científicos, decide ir más allá de lo que «el poder celestial permite». El eco de las palabras finales del Coro se percibe siglos después en los castigos de los sabios Frankenstein y Jekyll:

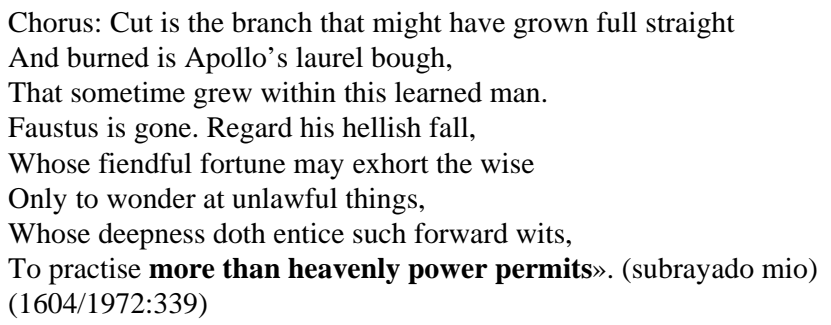

\section{FRANKENSTEIN: EL NOMBRE Y LA CRIATURA}

«It was the secrets of heaven and earth that I desired to learn», confiesa Victor Frankstein al capitán Walton al comienzo de la narrativa de su historia. Una historia que el primero quiere que sirva de advertencia al segundo para que no lleve a cabo su búsqueda a toda costa de lo desconocido, de las «unexplored regions» que se propone 
explorar. Vemos una vez más la fuerza de la ambición descubridora — científicaque arde dentro del alma humana. Como el Doctor Fausto, el Doctor Victor Frankenstein va a seguir su llamada imperiosa, va a traspasar los límites. Y va a ser castigado de forma implacable por ello. La investigación de Frankenstein, en este caso, se circunscribe fundamentalmente a la ciencia médica, inserta a su vez en lo que se llamó durante largo tiempo «Filosofía Natural»: «Natural Philosophy is the genius that has regulated my fate» (1818/1979:24).

Tras su partida a Ingolstadt para seguir sus estudios, el joven ginebrino Victor Frankenstein entra en contacto con M. Waldman, el profesor de Filosofía Natural que con sus métodos innovadores prenderá la mecha de la ambición científica de su alumno y marcará el rumbo de sus febriles investigaciones. Descubrir el principio de la vida será a partir de ahora su obsesión: la anatomía y la química serán las ramas de la Filosofía Natural que podrán darle a Frankenstein las respuestas que busca.

Para descubrir el principio de la vida, Victor Frankenstein estudia en profundidad la anatomía humana, se convierte en asiduo visitante de cementerios y depósitos de cadáveres. La muerte, reconoce pronto el joven doctor, va a serle imprescindible en su investigación. Los cuerpos de los muertos le ayudarán a descubrir qué es la vida, dándole a él la posibilidad de crearla a partir de la materia inerte e incluso descompuesta. El doctor Frankenstein no se conforma con curar enfermedades; él, como Dios, creará vida y dará vida a los muertos. Su entusiasmo se convierte en éxtasis, lo que atestiguan sus propias palabras:

\footnotetext{
«No one can conceive the variety of feelings which bore me onwards, like a hurricane, (subrayado mío) in the first enthusiasm of success. Life and death appeared to me ideal bounds, which I should first break through, and pour a torrent of light into our dark world. A new species would bless me as its creator and source; many happy and excellent natures would owe their being to me. No father could claim the gratitude of his child so completely as I should deserve theirs. Pursuing these reflections, I thought that if I could bestow animation upon lifeless matter, I might in process of time renew life where death had apparently devoted the body to corruption.» (1918:1979:39)
}

Impelido, como él mismo afirma, por la fuerza de un huracán, el joven doctor lleva a cabo sus investigaciones de forma obsesiva, lo que le hace recluirse, buscar en todo momento la soledad necesaria para llevar a cabo su trabajo. Porque, no lo olvidemos, los grandes genios científicos transgresores que configuran el arquetipo literario son grandes individualistas. Su trabajo no se lleva a cabo en colaboración. Sólo ellos están dispuestos a traspasar la frontera moral y a afrontar las siempre imprevisibles consecuencias. Frankenstein, además, irá progresivamente alejándose de aquellos que le aman y a los que él ama. Su amada Elizabeth, a quien Victor llamaba «my more than sister», su padre, amigos. Todos aquellos que han significado algo fundamental en su vida van siendo abandonados por el joven doctor, ya que éste se haya consumido por y sumido en su propósito científico: 


\begin{abstract}
The summer months passed while I was thus engaged, heart and soul, in one pursuit. It was a most beautiful season ... but my eyes were insensible to the charms of nature. And the same feelings which made me neglect the scenes around me caused me also to forget those friends who were so many miles absent, and whom I had not seen for so long a time. I knew my silence disquieted them ... (1818:1979:39-40).
\end{abstract}

La febril actividad física y mental de Frankenstein va dejando también huella en su aspecto, su palidez se hará evidente, sus mejillas se hundirán y sus ojos mostrarán pronto la expresión exaltada de la locura:

\footnotetext{
My eyeballs were starting from their sockets in attending to the details of my employment ... My cheek had grown pale with study, and my person had become emaciated with confinement ... (1818/1979:39).
}

Aunque el joven doctor es consciente del deterioro de su aspecto y de sus nervios, como también lo es de su alejamiento de sus seres queridos y de la preocupación que esto les acarrea, ni su salud ni sus vínculos afectivos son motivo suficiente para que él deje de perseguir su meta, lo que por fin, y para su horror, consigue en la famosa noche de noviembre, «It was on a dreary night of november that I beheld the accomplishment of my toil» (1818:1979:42).

A partir de este momento, el doctor Frankenstein será, como Dios, un creador. Pero la criatura a la que acaba de insuflar el principio de la vida - su descubrimiento secreto-, formada con restos de diferentes cadáveres humanos, no es hermosa como él había intentado hacerla, sino horripilante. Al contemplar al ser al que acaba de hacer vivir el doctor huye horrorizado: aquí comenzará la persecución del creador por su criatura, del padre-dios por su hijo monstruoso. A partir de aquí se unirán ineludiblemente los destinos de ambos hasta tal punto, y esto es significativo, que en la imaginación popular el monstruo creado por Frankenstein será conocido como «Frankenstein». La criatura rechazada, odiada, despreciada por su hacedor, sin nombre alguno en la novela de Mary Shelley, se adueñará del nombre de aquél que le dio vida. Encontramos aquí lo que parece ser una suprema metáfora de lo monstruoso: la transgresión monstruosa del científico producirá un monstruo real que le robará el nombre al primero, si bien en un plano extratextual.

El doctor y el producto viviente de su investigación están irremediablemente solos. El camino emprendido por Frankenstein, como el de Fausto, y, como se verá más adelante, el del doctor Jekyll, no tiene vuelta atrás. No existe posibilidad para estos Prometeos de la ciencia de recuperar su humanidad perdida, porque queriendo trascender las limitaciones humanas, queriendo ser más que humanos, se han convertido en monstruos, y su vida como tales ya no encuentra acomodo entre los hombres.

Mary Shelley escribió Frankenstein or the Modern Prometheus en plena época romántica. No cabe duda de que su novela y su protagonista son productos del Romanticismo. Pero se trata de un Romanticismo que si bien le debe mucho a la época 
en que se fraguó y tuvo su esplendor -último tercio del siglo dieciocho y primero del diecinueve- consiste fundamentalmente en una postura vital e intelectual que aunque encuentra terreno fértil en el momento histórico mencionado, trasciende las barreras temporales en la violencia de su imaginación y lo titánico de sus héroes. En este sentido, se puede decir que al igual que Frankenstein, son románticos el doctor Fausto, protagonista de una tragedia isabelina y, por tanto, ajena en el aspecto temporal al Romanticismo, y el doctor Jekyll, protagonista de una novela que se inserta en el último período victoriano. Los tres son héroes románticos en lo extremo de su empresa que les lleva a traspasar las barreras éticas y a situarse al otro lado de la ley. Como los héroes de Lord Byron o de Espronceda - Caín, el Pirata, o el Reo de Muerte- son románticos en su soledad, en su desarraigo. En relación con el siempre controvertido tema del Romanticismo, baste citar algunas obras seminales que mostraron nuevas posibilidades interpretativas y que, a la vez, atestiguan la complejidad de los términos «romántico» y «Romanticismo»: The Romantic Imagination 1949, de C.M.Bowra, Romanticism Reconsidered 1963, de N. Frye, The Roots of Romanticism 1965, de I. Berlin, 'German and Western Romanticism' en The Social History of Art, de A. Hauser 1951/73. Ilustrativos también a este respecto son los más recientes The Romantic Movement, 1994, de M. Cranston, Romanticism, Pragmatism, and Deconstruction 1993, de K. Wheeler, y Romantic Writings, 1996, de S. Bygrave (ed). Ya dijo Charles Whibley que la Odisea en su «very texture and essence» es una obra romántica, pero que en realidad el Romanticismo nació ya en el jardín del Edén y que la serpiente «was the first romantic» (en Bowra 1949:15).

En lo que respecta a la ciencia propiamente dicha, conviene resaltar el interés que numerosos escritores del período romántico sintieron por las investigaciones científicas que, por un lado, arrojaban nueva luz sobre la fisiología y la psique humanas y, por otro, parecían ofrecer nuevas posibilidades que para la mente romántica tenían que ser por fuerza subyugantes. Tras los fallidos intentos, en el siglo dieciocho, de Benjamin Franklin de curar la parálisis con electrochoques, Galvani abre nuevas esperanzas mostrando que la electricidad puede generar movimiento en un ser interte. El doctor Erasmus Darwin, cuenta la misma Mary Shelley, investigaba el principio de la vida manteniendo unos vermicelli en un frasco que, en un momento determinado, comenzaron a moverse. Es bien conocido el interés que Lord Byron y P.B. Shelley sentían por los experimentos de reputados científicos, y es de nuevo Mary Shelley quien da testimonio de esto en el prefacio que escribió para su famosa novela:

Many and long were the conversations between Lord Byron and Shelley to which I was a devout but nearly silent listener. During one of these, various philosophical doctrines were discussed, and amongst others the nature of the principle of life, and whether there was any probability of its being discovered and communicated. They talked of the experiments of Dr. Darwin ... who preserved a piece of vermicelli in a glass case till by some extraordinary means it began to move with voluntary motion. Not thus, after all, would life be given. Perhaps a corpse would be reanimated; galvanism had given token of such things, perhaps the 
component parts of a creature might be manufactured, brought together, and endued with vital warmth. (1818/1979:xiv)

Claro está, no son sólo las mentes de Byron y P.B. Shelley las que se excitan discutiendo estas perturbadoras posibilidades científicas. Mary Shelley es quien plasmará su indudable interés científico en su conocida novela y quien, como comenta J. Lamarca (1983:29), «evita la descripción minuciosa de este proceso científico, pero la alusión que de él nos hace permite entenderla como una especie de anticipación, una bioquímica avant la lettre». Como afirma R.D. Spector, «She (Mary Shelley) found what she needed in the awakened scientific and humanitarian interests of her age.» (Introduction to Frankenstein: «Mary's Monster» 1979:vii).

Es la mente de la jovencísima Mary Shelley la que, azuzada por estas perspectivas, creará a uno de los científicos más emblemáticos de todos los tiempos, el doctor Frankenstein, padre de una criatura sin nombre que en la imaginación popular se adueñará del nombre de su hacedor. La criatura que crea Victor Frankenstein presenta un aspecto monstruoso que no es sino reflejo de lo monstruoso de la acción del joven doctor que le ha dado la vida. Es víctima de un padre que le rechaza y aborrece desde el mismo momento de su nacimiento, es víctima del rechazo de los hombres que, rechazándole a él, rechazan lo monstruoso de su concepción. Mary Shelley, con espíritu humanitario, nos muestra la victimización de la criatura, que termina convirtiéndose en verdugo: una de sus víctimas será el propio Frankenstein, que ve cómo su hermano pequeño y su amada Elizabeth mueren a manos de la criatura que él ha creado. Las consecuencias de una acción transgresora no sólo son monstruosas, sino incontrolables. Esto nos dice esta suprema parábola de la transgresión en la que el doctor Frankenstein es verdugo y víctima de aquél a quién él victimiza por el hecho de crearle, de una criatura que no es otra cosa que la prolongación de sí mismo - paradigma de esa dualidad humana que de la misma forma indagará R.L. Stevenson años después a través de su doctor Jekyll-. Frankenstein y su criatura son inseparables. Sus nombres, como su destino, están unidos irremediablemente.

\section{DR. JEKYLL: LA PUERTA ABIERTA AL OTRO}

En la novela de R.L. Stevenson nos encontramos con una indagación en la duplicidad de la naturaleza humana. Lo que en un principio iba a ser tan sólo un thriller, esa primera versión que Stevenson escribió en tres días y que, al no satisfacer a su esposa Fanny, quien exigía mayores y más profundas implicaciones, él arrojó al fuego, se convertiría, a través del trágico personaje del doctor Jekyll, en paradigma de la dualidad y del poder destructivo del mal.

Es, sin duda, digno de consideración el hecho de que Jekyll sea un hombre de ciencia. Doctor en medicina, investiga las transformaciones fisioquímicas del cuerpo 
humano, y ha convertido el teatro de operaciones que se encuentra anexo a la casa que el doctor compró a los herederos de un cirujano, en un laboratorio, «... his own tastes being rather chemical than anatomical». Los experimentos de Jekyll tienen directa relación con la idea que ya expusiera Humphrey Davy en 1802 en su Discourse Introductory to a Course of Lectures on Chemistry. Según Davy, la química es «la parte de la filosofía natural que relaciona aquellas íntimas acciones de los cuerpos unos con otros, por las cuales se alteran su apariencia y se destruye su individualidad» (ver Crowther 1935:9). Conocido en la casa como laboratory o dissecting room, el lugar donde el doctor Jekyll lleva a cabo sus investigaciones se convertirá, como en el caso del doctor Frankenstein, en lugar de aislamiento. Cuando Mr. Utterson, el amigo abogado de Jekyll, pone allí el pie por primera vez, su mirada percibe lo sombrío e inquietante del lugar, visitado antes asiduamente por los estudiantes del cirujano:

It was the first time that the lawyer had been received in that part of his friend's quarters; and he eyed the dingy windowless structure with curiosity, and gazed round with a distasteful sense of strangeness as he crossed the theatre, once crowded with eager students and now lying gaunt and silent, the tables laden with chemical apparatus, the floor strewn with crates and littered with packing straw, and the light falling dimly through the foggy cupola. $(19: 22)$

El científico, que de forma habitual permanece largas horas encerrado en su laboratorio, irá recluyéndose aquí de forma progresiva a medida que se va produciendo su también progresiva deshumanización. Jekyll, consciente del carácter dual de su propia naturaleza, sabedor también de que la dualidad es inherente al ser humano, ensaya en su persona una droga compuesta por él mismo capaz de disociar de forma total los dos elementos opuestos que coexisten en su interior. El doctor Jekyll, hombre de cierta edad, sabio y respetable, aunque con algunas tendencias disolutas y propenso a placeres no muy edificantes, tras tomarse su propio brebaje experimentará alteraciones fisioquímicas que le convertirán en otro ser tan opuesto a él en temperamento como en aparencia. Sus dos personalidades, por medio de sus métodos científicos, se escinden dando lugar a dos criaturas diferentes, el doctor Henry Jekyll de siempre con su aspecto venerable y su elevada estatura, y el siniestro Edward Hyde, pequeño, ágil, joven, de aspecto tan repulsivo como amedrentador.

Tras tomar por primera vez su droga, el doctor Jekyll pasa por tremendos sufrimientos físicos a medida que su persona se transforma, pero, tras la agonía de la metamorfosis, tiene, ahora en la piel de la nueva criatura, una exultante sensación de vitalidad, de fuerza, de total despreocupación. Libre de las ataduras morales que sujetan a toda persona y la impiden llevar a cabo determinadas fechorías, el doctor Jekyll, ahora ya convertido en Hyde, disfruta de una libertad desconocida por él hasta este momento. La sensación es «dulce», como él mismo afirma en su confesión final, si bien su instinto es malvado y criminal. 


\begin{abstract}
... Then these agonies began swiftly to subside, and I came to myself as if out of a great sickness. There was something strange in my sensations, something indescribably new, and from its very novelty, incredibly sweet. I felt younger, lighter, happier in body; within I was conscious of a heady recklessness, a current of disordered sensorial images running like a mill race in my fancy, a solution of the bonds of obligation, an unknown but not an innocent freedom of the soul. I knew myself, at the first breath of this new life, to be more wicked, tenfold more wicked, sold a slave to my original evil; and the thought, in that moment, braced and delighted me like wine (19:50).
\end{abstract}

Lo «dulce» de la sensación de libertad exenta de principios éticos para dar rienda suelta a los instintos más perversos es, ciertamente, algo perturbador, como también lo es el hecho de que el doctor Jekyll, una vez realizado su experimento, decida sacar provecho de su nueva persona y realizar como Mr. Hyde todo tipo de actos criminales con la segura impunidad de cambiar a voluntad de aspecto y escapar a la justicia bajo la apariencia de ejemplar ciudadano. La ciencia hará esto posible. La composición química largo tiempo estudiada hará de Jekyll también un dios. Como ya hicieran Fausto y Victor Frankenstein, Jekyll traspasa los límites morales de la mano del dominio científico. Como Frankenstein y su criatura, el doctor Jekyll creará un monstruo a partir de sí mismo del que ya nunca podrá escapar. Jekyll, seguro en un principio de que puede controlar su invento, utiliza su brebaje ya para ser Jekyll, ya para ser Hyde, sacando partido de ambos estados, adormeciendo su conciencia cuando es Jekyll, buscando el aspecto libre de toda sospecha del doctor Jekyll cuando es Hyde y, aunque carente de todo arrepentimiento, teme ser ajusticiado por asesinato. Pero como sucede en los casos de Fausto y Frankenstein, el control es tan sólo una ilusión. Ninguno de los tres científicos, una vez dado el paso transgresor, es capaz de controlar la situación. Las consecuencias de la misma son de nuevo incontrolables, imprevisibles.

En la novela de Stevenson existe un elemento que, a mi entender, además de cumplir su función en el engranaje de la trama actúa como poderosa metáfora: la puerta. Ya en el primer capítulo de la novela es una puerta la que ostenta protagonismo tal y como atestigua su título, «Story of the Door». Es una puerta la que trae a la memoria del señor Enfield lo que cerca de ella sucedió teniendo como protagonista al siniestro Edward Hyde. A partir de aquí vemos cómo el doctor Jekyll, tras haber abierto la puerta (esta vez hablando metafóricamente) a su otro yo con su brebaje, abre todas las puertas de su casa a Hyde quien, como es lógico, puede entrar en y salir del laboratorio siempre que lo estime oportuno o necesario. El mal que se hallaba encerrado y controlado dentro de los confines del cuerpo de Jekyll se escapa, gracias a su saber científico, y toma cuerpo en Hyde. Es la droga de Jekyll, junto con sus deseos inconfesables, la que abre la puerta al mal. Y el mal, como sucede con Fausto y con Frankenstein, como sucede en las grandes tragedias, una vez que queda en libertad, cuando se le abre la puerta, es una fuerza incontrolable que se apodera de todo lo demás. 
El sólido y potente cerrojo que Jekyll se hace instalar en la puerta de su laboratorio para cerrar literalmente la puerta a su tentación resulta ser ineficaz. El doctor Jekyll intenta, ya tarde, cerrar la puerta a Hyde dejando definitivamente de tomar su droga. Pero la fuerza de Hyde es tal, que aparece sin necesidad de que Jekyll la tome: el cuerpo del doctor se convierte cuando él menos lo espera y menos lo desea en el monstruoso cuerpo de Hyde, nombre que recrea la idea de lo oculto, lo escondido, a través del verbo homófono inglés to hide (esconder, ocultar). Una vez que Hyde toma cuerpo y sale a la luz, terminará por no poder esconderse ya en el cuerpo de Jekyll porque será más poderoso que éste, porque el mal traspasó la puerta. Jekyll se la abrió. La ciencia, en manos de un doctor como Jekyll, puede ser una puerta abierta a la destrucción.

\section{CIENCIA Y LITERATURA: DOS CARAS DE UNA BÚSQUEDA}

Considero de gran importancia subrayar el hecho de que la ciencia y la literatura se hallan mucho más cerca de lo que comúnmente pueda creerse. Hay dos cuestiones básicas que muestran la existencia de esta larga y estrecha relación. Primeramente, la ciencia nunca le ha sido ajena a la literatura. La ciencia y el científico han sido con frecuencia tema y motivo literarios, y los tres doctores protagonistas de este estudio han consolidado nada menos que todo un arquetipo en la historia de la literatura. La segunda cuestión, que puede que sea incluso origen de la primera, es que los dos ámbitos, el científico y el literario o, si se prefiere, ambas actividades, parecen ser con toda probabilidad dos manifestaciones de un impulso común: el deseo de explicar o explicarse el mundo. El deseo de entender la realidad, de todo eso que llamamos vida. A menudo se piensa que el misterio se encuentra en lo sobrenatural, lo que no pongo en duda, pero lo natural es el misterio. Aquello que tocamos, que vemos, que percibimos con los sentidos, tiene una existencia que, como la nuestra propia, sigue siendo un misterio tanto para la ciencia como para la literatura: ambas, cada una a su manera, pugnan por descubrirlo. La vida sigue siendo un enigma para la ciencia y para la literatura, lo que ha hecho que tanto científicos como literatos hayan aportado contribuciones de extraordinario valor a lo largo de la historia. La historia de la ciencia, como la de la literatura, lleva escribiéndose durante siglos y ambas han consolidado un canon que, aunque deba estar abierto a cualquier revisión o ampliación, está marcando pautas fundamentales en ambos campos.

El que la literatura se haya interesado por la ciencia y, consecuentemente, por algunos de sus representantes - astrónomos, biólogos o médicos, entre otros- es algo que no debe sorprendernos si tenemos en cuenta que la búsqueda apasionada del científico, su lucha con el entorno y hasta con determinados principios éticos como en los tres casos que acabamos de mostrar, es algo eminentemente literario en el sentido de que la literatura — que es otra forma de indagación— lo reconoce como suyo. 
Cuando un escritor hace del científico el protagonista de su obra, incluso cuando el tema de la misma es la ciencia, comprobamos que casi nunca existen descripciones detalladas de los procesos de investigación, que no se explica científicamente en qué consiste determinado avance revolucionario. El literato no es un hombre de ciencia y carece, salvo quizá alguna excepción, de la formación necesaria en este sentido para manifestarse de forma explícita. Elude la explicación y suele recurrir a la elipsis. Es su forma, perfectamente lógica, de tratar estas cuestiones. Pero lo importante es el hecho de que el buen escritor, aun sin dominar, incluso sin apenas conocer las disciplinas científicas, consigue hacer creíbles su tema y su protagonista. George Eliot nos muestra a un doctor Lydgate del todo convincente. Al igual que Thomas Hardy con su doctor Fitzpiers, o incluso en los casos extremos, arquetípicos, Mary Shelley y R. L. Stevenson realizan retratos apasionantes y conmovedores de sus médicos prometeicos. $\mathrm{La}$ empatía, constituyente del talento literario, hace que esto sea posible. La empatía entre quien escribe y quien investiga en cualquier parcela de la ciencia. Porque ambos, literato y científico, son, a su manera, creadores. Aunque no sobrepasen los límites éticos como Fausto, Frankenstein o el doctor Jekyll, literatos y científicos comparten cierta vocación fáustica en su deseo de agrandar las fronteras de lo real: «Estoy en la antesala de los misterios», decía Pasteur en una frase reveladora, «y el velo que los cubre se vuelve cada vez más fino» (ver Koestler 1977:9). Esto mismo pensaron Fausto, Frankenstein y Jekyll en algún momento de su investigación. Los poetas y escritores en general seguramente experimentan algo parecido.

Esta búsqueda, este afán por descubrir el misterio, este ir siempre hacia adelante, han contribuido de forma portentosa, sobre todo en el campo científico, a nuestra supervivencia, a nuestra calidad de vida. Pero siempre parece permanecer sin contestar la cuestión de hasta dónde se puede seguir, qué límites pueden o no traspasarse, si se puede separar la investigación de su aplicación tecnológica. El libro de R. Shattuck, antes aludido, es un ejemplo de esta profunda preocupación, y a lo largo de un exhaustivo e interdisciplinar análisis ahonda en este dilema:

En un número de la revista Daedalus dedicado a 'Los Límites de la Ciencia' (Primavera 1978), el ensayo del biólogo molecular David Baltimore es un enérgico alegato contra cualquier limitación de la ciencia pura. Baltimore no coincide con Bacon ni reconoce dificultad alguna para separar la cabeza del cuerpo de la Esfinge. 'Quiero hacer una distinción crucial. Los argumentos (a favor de una libertad ilimitada) atañen a la investigación científica pura, no a las aplicaciones tecnológicas de la ciencia. Al pasar de lo puro a lo aplicado mis argumentos pierden valor'. ¿Pero podremos alguna vez trazar una divisoria clara -o incluso tosca- entre descubrimiento y aplicación? Y si podemos, ¿tendríamos que sostener que los científicos responsables deben permanecer en el lado puro de la divisoria? (Shattuck 1998:219)

Fausto, Frankenstein y Jekyll fueron científicos transgresores. Su transgresión les dio una dimensión monstruosa y les valió un castigo implacable, pero a la vez les dio una dimensión arquetípica y trágica. Más allá del planteamiento sólo a primera vista 
moralizante de estas obras está el resbaladizo terreno de la ambivalencia. Estos tres grandes científicos de ficción, estos tres arquetipos literarios siguen todavía vivos. Su vigencia puede verse reforzada en una actualidad como la nuestra en la que los enormes avances científicos como los relacionados con la ingeniería genética, entre otros de gran trascendencia, abren nuevas interrogantes, reclaman debates éticos, suscitan temores, generan esperanzas y, por supuesto, están dando ya sus frutos en el campo de la literatura.

\section{BIBLIOGRAFÍA}

BERLIN, I. (1965), The Roots of Romanticism, Princeton University Press, Princeton.

BOWRA, C.M. (1949), The Romantic Imagination, Harvard.

Bygrave, S. (ed) (1996), Romantic Writings, London, Routledge.

Cranston, M. (1994), The Romantic Movement, Oxford, Blackwell.

CROWTHER, J. G. (1935), British Scientists of the 19th Century, London, Routledge.

ELIOT, G. (1872/1976), Middlemarch, Penguin, Harmondsworth.

FRYE, N. (1963), Romanticism Reconsidered, Columbia University Press.

HARDY, T. (1887/1982), The Woodlanders, Penguin, Harmondsworth.

HAuser, A. (1951/1973), The Social History of Art, vol. 3, London, Routledge \& Kegan Paul.

Koestler, A. (1977), The Act of Creation, Picador, Pan Books.

Lamarca Margalef, J. (1983), Ciencia y Literatura. El Científico en la Literatura Inglesa de los siglos XIX y XX, Ediciones de la Universidad de Barcelona, Barcelona.

Mangan, M. (1989), Doctor Faustus, (Penguin Critical Studies), Penguin, Harmondsworth.

Marlowe, Ch. 1604/1972, Dr. Faustus en Christopher Marlowe: The Complete Plays, Penguin, Harmondsworth.

Shattuck, R. (1998) Conocimiento Prohibido. De Prometeo a la Pornografía.Trad. Eva Rodríguez Halfter, Madrid, Taurus.

SHELley, M. 1818/1979, Frankenstein or the Modern Prometheus, Bantam Pathfinder, New York.

SPECTOR, R.D. (1979), Introduction to Frankenstein, «Mary's Monster», en M. Shelley: Frankenstein, Opus Cit.

Stevenson, R. L. 1886/1993, The Strange Case of Dr. Jekyll and Mr. Hyde, Wordsworth Classics, Hertforthshire.

Thorlby, A.K. (1969), The Romantic Movement, Longmans, London.

WhEeLER, K. (1993), Romanticism, Pragmatism and Deconstruction, Oxford, Blackwell.

White, M. (1985), «Dr. Lydgate: the literary characterization of the Doctor at a Historical Turning Point», Asclepio, XXXVII, pp.321-349. 